\title{
Compresión del nervio cubital en codo por condromatosis sinovial primaria
}

\author{
Ulnar nerve compression at the elbow by \\ primary synovial chondromatosis
}

Claudia Arroyo Berezowsky, ${ }^{\star}$ Eduardo Hernández Méndez Villamil, ${ }^{\ddagger}$ Alejandro Espinosa Gutiérrez§

\begin{abstract}
RESUMEN
La compresión del nervio cubital en el canal cubital es la segunda neuropatía compresiva más frecuente en la extremidad superior. El sitio de compresión más habitual es en el codo, en el área del ligamento de Osborne. La condromatosis sinovial es una lesión benigna, primaria, que consiste en masas nodulares de cartílago hialino dentro de la membrana sinovial de una articulación. Es común en articulaciones diartrodiales de carga y la localización más común se da en la rodilla. Se han reportado casos de sinovitis condromatosa en el codo, pero se considera una zona poco frecuente. A continuación, se presenta el caso de una mujer de 72 años de edad con antecedente de luxación de codo izquierdo, quien mostró síntomas de doble compresión del nervio cubital en codo y en muñeca. La compresión del nervio en el codo resultó ser por una causa poco habitual: una condromatosis sinovial primaria diagnosticada por patología, que inicialmente se tomó por formar calcificaciones heterotópicas.
\end{abstract}

Palabras clave: Nervio cubital, condromatosis sinovial, codo, síndrome de canal cubital.

Nivel de evidencia: IV

\footnotetext{
* Cirujana de Mano y Ortopedista del Centro Médico ABC.

‡ Cirujano de Mano y Ortopedista. Médico adscrito al Servicio de Mano del Instituto Nacional de Rehabilitación.

$\S$ Cirujano de Mano y Ortopedista. Jefe del Servicio de Mano del Instituto Nacional de Rehabilitación.
}

Recibido para publicación: 07/01/2020. Aceptado: 15/03/2020.

Correspondencia:

Dra. Claudia Arroyo Berezowsky

Av. Vasco De Quiroga Núm. 4299, consultorio 1003, Col. Lomas de Santa Fe, 05348, Cuajimalpa de Morelos, CDMX.

Tel: (55) 55402040

E-mail: dra.carroyob@gmail.com

\begin{abstract}
Ulnar nerve compression in the cubital tunnel is the second most frequent compressive neuropathy in the upper extremity after median nerve compression in the carpal tunnel. Primary synovial chondromatosis is a benign lesion consisting on nodular masses of calcified hyaline cartilage inside the synovial membrane of a specific joint. It is more common on diarthroideal, weight bearing joints, and the most common site of presentation is the knee. Primary synovial chondromatosis has been reported around the elbow, but it is considered a rare location. We present the case of a 72 year old woman with a history of left elbow dislocation, who presented with ulnar nerve compression symptoms at the elbow and wrist. The elbow compression turned out to be caused by primary synovial chondromatosis that was diagnosed with pathology. We initially believed the synovial chondromatosis was heterotopic calcification.
\end{abstract}

Keywords: Ulnar nerve, primary synovial chondromatosis, elbow, cubital tunnel syndrome.

Level of evidence: IV

\section{INTRODUCCIÓN}

La compresión del nervio cubital es la segunda neuropatía compresiva más común de la extremidad superior. Puede provocar dolor, parestesias o debilidad en la musculatura extrínseca e intrínseca de la mano. Existen diferentes sitios de posible compresión en el trayecto del nervio cubital como el codo y la muñeca. ${ }^{1,2}$

Aún se debate sobre el tratamiento óptimo de la compresión del nervio cubital. Hay cirujanos que creen que la historia natural de la compresión del nervio cubital tiende a una mejoría. Algunos autores consideran que sin la intervención quirúrgica, la compresión del 
nervio cubital puede resultar en cambios sensitivos y motores irreversibles con importante morbilidad asociada. Otros apoyan la teoría sobre la mejoría clínica de manera natural cuando hay síntomas leves. ${ }^{2}$

La condromatosis sinovial es una lesión benigna, primaria, donde hay proliferación del tejido sinovial y formación de cuerpos osteocondrales en una articulación. Se considera una patología autolimitada. Cuando es sintomática, tiene una presentación poco específica y, por lo general, se encuentra en articulaciones diartroideas de carga. La localización más frecuente es en la rodilla, seguida de la cadera, el hombro, el codo, el tobillo y la muñeca. ${ }^{3}$

En 2018, se presentó en el Servicio de Cirugía de Mano una paciente de 72 años de edad con historia de fractura-luxación de codo izquierdo, 10 años antes tratada en otro lugar. Mostraba dolor en codo, sensación de bloqueo articular, con déficit de $20^{\circ}$ de extensión, y parestesias en el territorio del nervio cubital de cuatro años de evolución. También manifestaba abducción involuntaria del meñique, dificultad para flexionarlo y debilidad de la mano izquierda. Negaba inestabilidad en codo izquierdo. A la exploración física inicial del codo se detectó flexión completa, un déficit de $20^{\circ}$ de extensión, sin evidencia de inestabilidad en varo o valgo. En la mano se observó hipotrofia de los músculos de la región hipotenar y primer espacio interóseo dorsal. El signo de Pollock fue positivo (debilidad en flexor carpi ulnaris, debilidad en flexor digitorum profundus del dedo meñique y anular), se encontraron también un signo de Wartenberg (abducción involuntaria del meñique) y signo de Froment positivo (flexión de falange distal del pulgar para retener un objeto entre los dedos, debido a la debilidad del músculo aductor del pulgar). Había debilidad para aducción y abducción de dedos.
Se detectó signo de Tinel positivo en el canal cubital, en el surco epitrócleo-olecraneano y en el canal de Guyon; también se detectó un signo de rascado y colapso positivo en canal cubital, hipoestesias en dedo meñique y lado cubital del dedo anular, con predominio en el dedo meñique. Clínicamente no había datos de radiculopatía cervical. Los estudios de electrodiagnóstico reportaron degeneración axonal motora y sensitiva de nervio cubital izquierdo y radiculopatía motora aguda de $\mathrm{C} 7$ y $\mathrm{C} 8$.

La Figura 1 muestra las radiografías preoperatorias del codo izquierdo, donde se observa deformidad del olécranon y de la cúpula radial. Se consideró que la deformidad en la superficie articular olecraneana era la causa de la sensación de bloqueo y limitación en arcos de movimiento del codo. También se pueden observar calcificaciones extraarticulares alrededor del codo. Al principio se pensó que eran osificaciones heterotópicas como consecuencia de la luxación previa del codo y se asumió que se trataba de un hallazgo radiográfico. No se consideró que tuvieran relación con los datos clínicos y no había plan para su resección.

El plan de tratamiento fue una descompresión quirúrgica abierta del nervio cubital en canal cubital y en canal de Guyon por haber sospecha de una doble compresión del nervio cubital izquierdo.

Al realizar el abordaje medial en el codo para la descompresión del canal cubital se detectó importante fibrosis alrededor del nervio cubital. Al liberar el nervio de la fibrosis, se observó una masa profunda al nervio cubital que lo desplazaba y comprimía. El nervio cubital se encontraba aplanado y pálido en su recorrido sobre la tumoración. Se observaron calcificaciones encapsuladas en un tejido que tenía continuidad con la cápsula posteromedial húmero-cubital en la salida del surco epitrócleo-olecraneano, proxi-
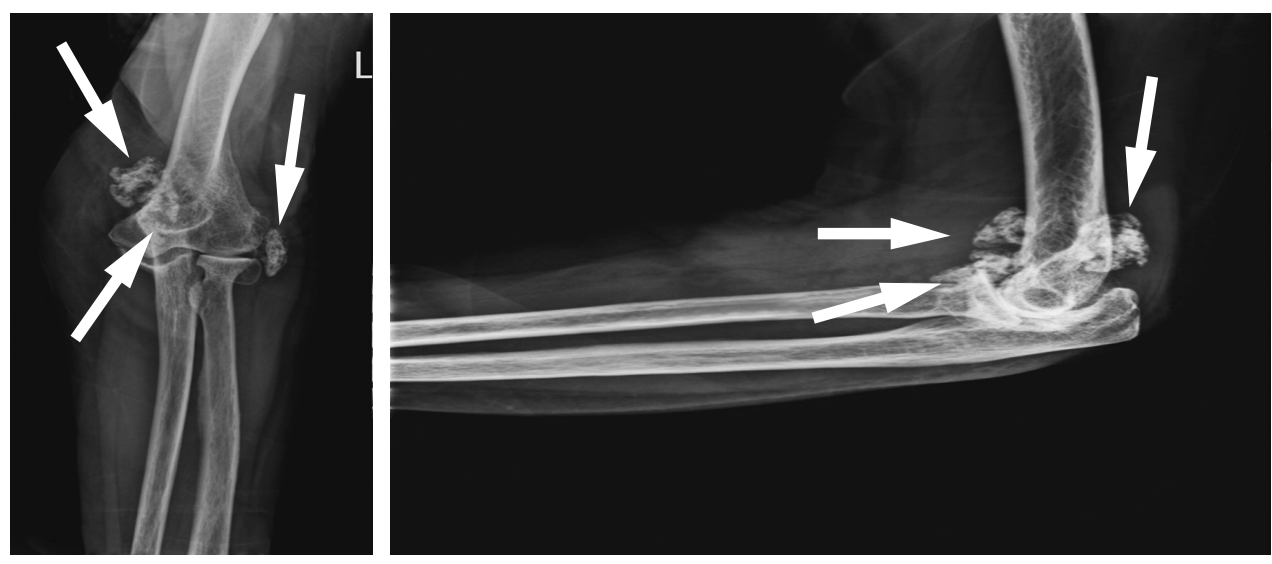

Figura 1:

Radiografías AP y lateral de codo izquierdo: se observa deformidad en cúpula radial y olécranon. Se aprecian tres masas (flechas blancas) con calcificaciones: una posteromedial y dos anterolaterales. 


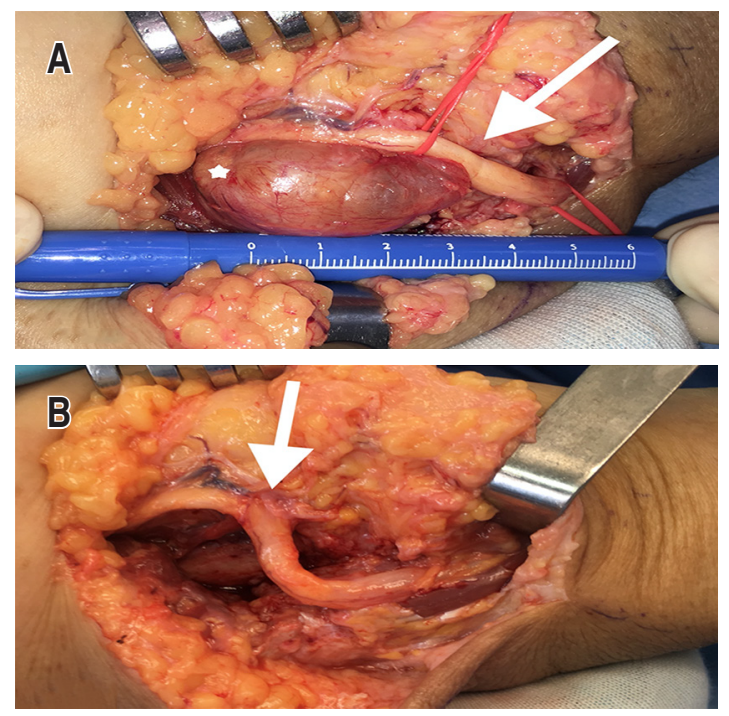

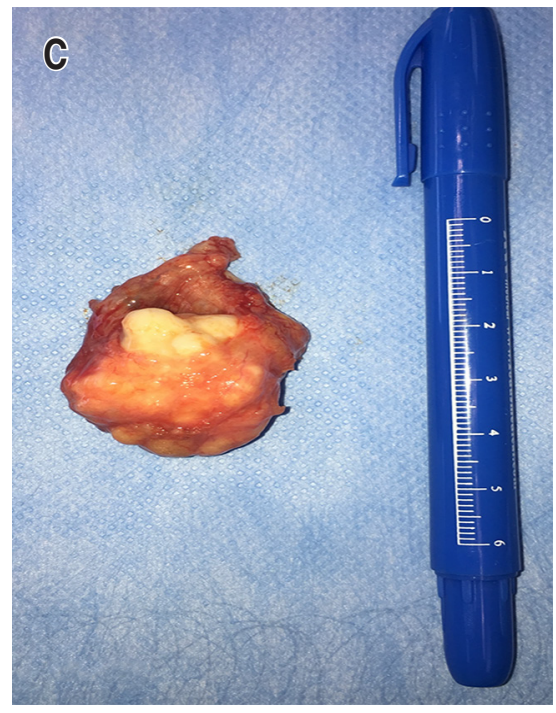

Figura 2:

Imagen transquirúrgica de la descompresión del nervio cubital en el codo. mal al ligamento de Osborne. Todo el saco contenía líquido viscoso, de apariencia similar al líquido sinovial y una masa de material amarillento, con nódulos calcificados de aproximadamente $4 \times 3 \times 2 \mathrm{~cm}$, que se resecó y envió a patología. Además, se liberó el nervio cubital desde la arcada de Struthers hasta el ligamento de Osborne y se realizó epineurólisis. La Figura $2 A$ muestra imágenes transquirúrgicas de la masa desplazando el nervio cubital, la masa resecada se puede observar en la Figura $2 B$ y la resultante tortuosidad del nervio después de la resección de la masa se muestra en la Figura $2 C$. Al terminar la resección se detectó un defecto capsular articular y el nervio cubital se volvió tortuoso. El nervio no se regresó al surco epitrócleo olecraneano. Al realizar movimientos de flexión y extensión, el nervio no migraba, por lo que no se efectuó ninguna estabilización del nervio con tejidos blandos. Después del cierre por planos se hicieron pruebas de estabilidad sin percibir inestabilidad en varo, valgo o extensión completa.

A continuación, se descomprimió el nervio cubital en el canal de Guyon. ${ }^{1}$ Se liberaron la zona 1, 2 y 3. En la zona 1 se detectó un nervio comprimido, aplanado y pálido. Al terminar el procedimiento quirúrgico no se dejó drenaje en el codo. Se aplicó un vendaje algodonoso y después de 10 días se retiró el material de sutura. Al no haber datos de inestabilidad transquirúrgica, se permitió movimiento sin restricción del codo. La paciente acudió al Servicio de Rehabilitación para mejorar arcos de movimiento del codo, así como reeducación motora y sensitiva.

En las Figuras $3 A$ y $B$ se muestran las radiografías postoperatorias del codo izquierdo. Se puede ob- servar que se resecó la masa posteromedial, pero aún se aprecian otras dos masas en el codo izquierdo. El reporte definitivo de patología para la masa del codo fue condromatosis sinovial.

Tres meses después del procedimiento, los síntomas sensitivos habían mejorado, presentaba menos parestesias y mejoría de la sensibilidad en la mano; sin embargo, refería sensación de inestabilidad del codo izquierdo que se comprobó con una prueba de estrés en valgo. Se envió a valoración por el especialista en codo para evaluar tratamiento para inestabilidad en valgo del codo izquierdo.

Después de siete meses del procedimiento quirúrgico, la paciente mostraba arcos de movimiento completos de codo sin sensación de bloqueo, ligero dolor en la cicatriz quirúrgica que mejoró con masaje y liberación de adherencias. La prueba de Tinel fue negativa en el codo y aún positiva en el canal de Guyon. La fuerza del flexor carpi ulnaris y flexor digitorum profundus del meñique mejoró junto con la fuerza de prensión. La mano izquierda persistía con menor fuerza que la derecha. La sensibilidad mejoró en los dedos meñique y anular, aunque aún era menor en la mano contralateral. Respecto a la inestabilidad de codo, en el servicio de codo no se consideró necesaria una estabilización quirúrgica del codo izquierdo, debido a la baja demanda funcional de la paciente. La paciente no acudió más a seguimiento.

\section{DISCUSIÓN}

La compresión del nervio cubital es la segunda neuropatía compresiva más común en la extremidad 
superior. ${ }^{1}$ El nervio cubital se dirige a través del compartimento anterior del brazo y al atravesar el septo intermuscular medial pasa al compartimento posterior. La arcada de Struthers es una banda aponeurótica que se encuentra proximal al canal cubital entre el epicóndilo medial y la aponeurosis flexorapronadora profunda. ${ }^{4}$

$\mathrm{Al}$ salir de la arcada de Struthers, el nervio pasa detrás del epicóndilo medial y entra al antebrazo por el canal cubital en el codo. El canal cubital está delimitado en la parte superior, a manera de techo, por el ligamento arqueado de Osborne. El ligamento va desde el epicóndilo medial a cabeza humeral de flexor carpi ulnaris hasta el olécranon y la cabeza cubital de flexor carpi ulnaris. El piso del túnel está formado por el ligamento colateral medial, cápsula y olécranon. ${ }^{4,5}$ Cuando el codo se flexiona, puede haber una excursión de $2.2 \mathrm{~cm}$ del nervio cubital y el diámetro del canal disminuye entre 30 y $40 \% .^{5}$ En el antebrazo, el nervio cubital transcurre profundo al flexor carpi ulnaris y superficial al flexor digitorum profundus. Al llegar a la muñeca, se vuelve lateral al flexor carpi ulnaris y medial a la arteria cubital. Aquí, el nervio cubital entra en el canal de Guyon. ${ }^{2,4,5}$ El canal de Guyon se divide en tres zonas anatómicas. La primera incluye la rama motora y sensitiva. La segunda zona contiene la rama motora profunda y la tercera tiene la rama superficial sensitiva del nervio cubital. ${ }^{2}$

El sitio de compresión más común del nervio cubital es en el codo bajo el ligamento de Osborne. Otros lugares de compresión pueden ser la cabeza medial del tríceps, la arcada de Struthers, la entra- da o salida a través del flexor carpi ulnaris, la aponeurosis flexora-pronadora y el canal de Guyon en la muñeca. . $^{1,4,5}$

Los síntomas y datos clínicos de compresión del nervio cubital varían dependiendo del sitio de compresión. Cuando existe compresión en el codo o en el antebrazo, los datos clínicos más comunes son: dolor, parestesias e hipoestesias en el lado cubital de la mano. En el caso de la compresión proximal al canal cubital, puede haber alteraciones en la sensibilidad en la rama cutánea sensitiva dorsal. ${ }^{5}$ Puede haber debilidad de los flexores extrínsecos de la mano como el flexor carpi ulnaris y flexor digitorum profundis $4^{\circ}$ y $5^{\circ}$, disminución de la fuerza de prensión, así como atrofia de los músculos intrínsecos de la mano. ${ }^{2}$

En la exploración física se puede apreciar debilidad de los interóseos, del adductor pollicis y lumbricales cubitales. El signo de Wartenberg (abducción del meñique), signo de Froment y mano en garra se encuentran en estadios avanzados de compresión del nervio cubital. La prueba de Tinel puede ser positiva al percutir sobre el sitio de compresión. ${ }^{5}$ La prueba de «rascado y colapso» descrita por Cheng y Mackinnon en 2008 demostró alta sensibilidad, incluso mayor que la prueba de Tinel, para el diagnóstico de compresión del nervio cubital en el codo (con una exactitud de 89\%). ${ }^{6,7}$

Identificar la zona de compresión del nervio cubital puede ser difícil y los diagnósticos diferenciales incluyen neuropatías compresivas como enfermedad cervical discal y neuropatías motoras. En estos casos, los estudios de electroconducción pueden ayudar a identificar el sitio de compresión. ${ }^{4}$ El diagnóstico
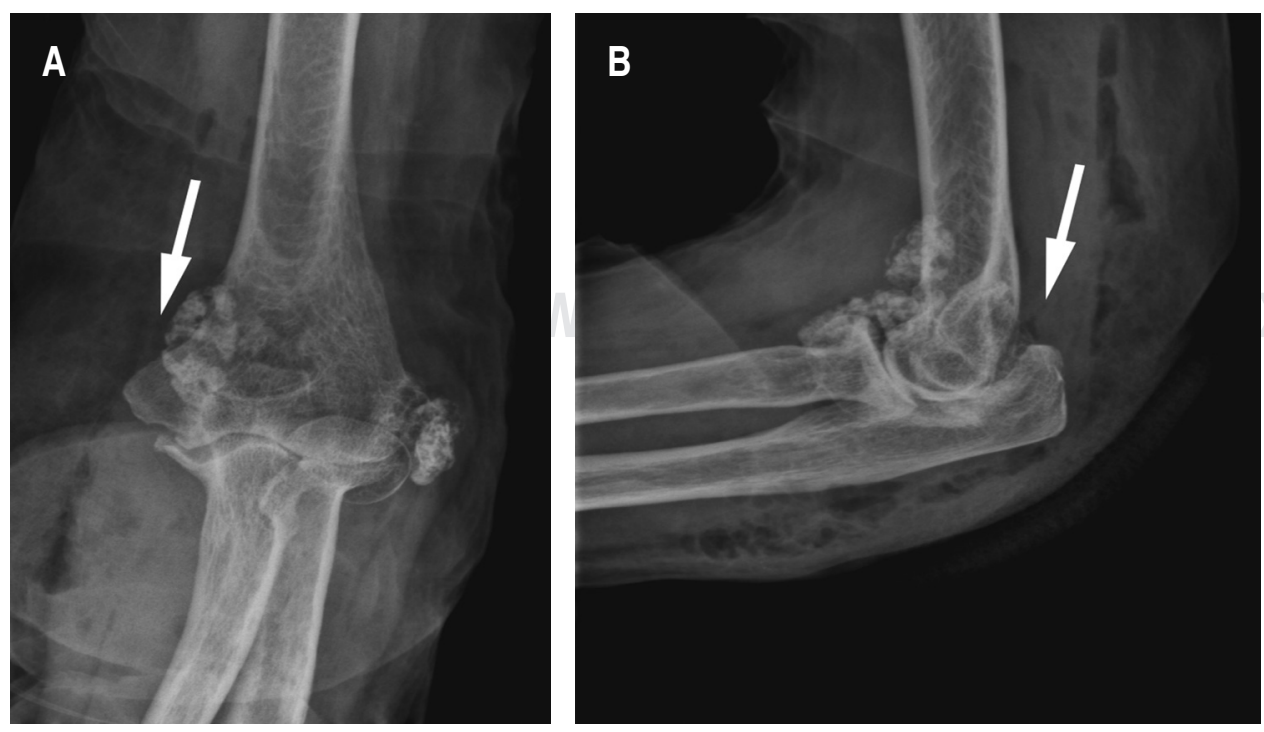

Figura 3: 
es particularmente complicado en los pacientes con sintomatología de compresión de nervio cubital multinivel y en aquéllos con datos no demostrables en estudios de electrodiagnóstico. ${ }^{1,8}$

En la actualidad, se considera que el tratamiento conservador de la compresión del nervio cubital es aceptable como manejo inicial para la mayoría de los pacientes. No existe suficiente evidencia que establezca el momento específico para realizar la descompresión quirúrgica. Tampoco se ha demostrado que una técnica quirúrgica sea superior a otra (descompresión sola o descompresión con transposición anterior del nervio cubital). ${ }^{9}$ Sin embargo, algunos autores defienden que si no se trata la compresión del nervio cubital de manera temprana, puede llegar a atrofia muscular y limitación funcional irreversible de la mano. ${ }^{1}$

En este caso clínico, la paciente tenía antecedente de una luxación de codo, presentaba datos clínicos de compresión de nervio cubital en canal cubital, pero también en el canal de Guyon y los estudios de electrodiagnóstico no contribuyeron a revelar el sitio de compresión. Las radiografías de codo mostraban calcificaciones periarticulares que se habían considerado osificaciones heterotópicas por el antecedente de la luxación del codo. La osificación heterotópica es una complicación conocida después de lesiones en el codo y la morfología de las lesiones puede ser variable. En un estudio por Foruria y colaboradores se observó que la osificación heterotópica interfería con el movimiento en $20 \%$ de codos tratados quirúrgicamente después de fracturas-luxaciones. De éstos, $10 \%$ requirieron tratamiento quirúrgico para resección de osificación heterotópica. ${ }^{10}$

En un inicio no se consideró que las calcificaciones fueran causa de la compresión del nervio cubital o del bloqueo articular. Se decidió realizar descompresión quirúrgica del nervio cubital, tanto en canal cubital como en el canal de Guyon. No se tenía contemplado resecar los cuerpos calcificados, ya que eran extraarticulares. Sin embargo, como se mencionó anteriormente, en el canal cubital, entre el surco epitrócleo-olecraneano y el ligamento de Osborne, se detectó una masa que desplazaba y comprimía el nervio cubital. Esta masa se resecó como parte de la descompresión del canal cubital.

El reporte de patología de la masa enviada fue de condromatosis sinovial. La condromatosis sinovial también se conoce como síndrome de Reichel. ${ }^{11} \mathrm{Es}$ una lesión poco común, benigna, caracterizada por formación sinovial de cuerpos cartilaginosos y osteocondrales, típicamente intraarticulares, aunque también puede ser extraarticular. Esta condición puede simular otras condiciones tumorales. Los datos clínicos son poco específicos. Puede haber disminución de los arcos de movimiento de una articulación, dolor, aumento de volumen y crepitación. ${ }^{3,11}$ Tanto la presentación primaria como la secundaria son poco comunes.

Por lo general, se presenta en articulaciones diartroideas en pacientes entre 30 y 60 años de edad, y es más común en hombres (1.8-tres veces más) que en mujeres. Las localizaciones más frecuentes son: rodilla (70\%), cadera (20\%), hombro, codo, tobillo y muñeca. Tradicionalmente, se ha descrito que esta condición afecta las articulaciones de carga y se acepta que mientras más peso recibe una articulación, mayor es el potencial para desarrollar condromatosis sinovial. ${ }^{3}$

La condromatosis sinovial fue descrita por Milgram como una patología autolimitada, y la clasificó en tres estadios desde el inicio hasta su resolución. Esta clasificación se basó en morfología macro- y microscópica. El estadio I representa un proceso intrasinovial inflamatorio activo sin cuerpos libres. El estadio II involucra la proliferación del tejido sinovial con cuerpos libres transicionales. El paciente puede mostrar datos clínicos de bloqueo mecánico. El estadio III se caracteriza por la presencia de múltiples cuerpos libres con mínima actividad inflamatoria sinovial. Estos pacientes pueden ser asintomáticos. ${ }^{3,12}$

En los estudios radiográficos se encontrarán imágenes esferoideas, con un borde externo más calcificado que el centro, que serán radiolúcidas, multifocales, articulares o periarticulares. Estas imágenes se consideran patognomónicas de la condromatosis sinovial. ${ }^{3}$ Se debe tomar en cuenta que la calcificación de las esferas depende del tiempo, por lo que aproximadamente $20 \%$ de los casos (estadio I) no tienen evidencia de calcificaciones en los estudios radiográficos. En estos casos, se pueden requerir estudios de imagen avanzados como resonancia magnética o tomografía axial computarizada. ${ }^{3}$

El diagnóstico diferencial por imagenología incluye osteocondritis disecante, artrosis con cuerpos libres, tuberculosis, artropatías hemopáticas, fracturas osteocondrales, seudogota con calcificaciones, tumores de tejidos blandos y otros tumores sinoviales benignos. ${ }^{3,12}$

Se considera que la condromatosis sinovial es un padecimiento autodelimitado y benigno, por lo que en estadios iniciales ${ }^{1}$ puede intentarse un manejo conservador con analgésicos y antiinflamatorios. No obstante, el tratamiento quirúrgico está indicado si existe bloqueo articular o síntomas mecánicos. Actualmente, no existe evidencia que apoye que la 
resección quirúrgica de los cuerpos libres intraarticulares con sinovectomía sea mejor que la resección simple de éstos. Tampoco se ha encontrado evidencia que demuestre que ninguno de los dos procedimientos disminuya la tasa de recurrencia. Lo que se ha observado es que el sinovio con evidencia histológica de condrometaplasia tiene mayor riesgo de recurrencia. La tasa de recurrencia con resección de cuerpos libres es de alrededor de 3 a $60 \%$ y la recurrencia con sinovectomía total se ha reportado en $0-8 \% .{ }^{1}$ La tasa de recurrencia en artroscopia de cadera se ha reportado hasta en $7.1 \%$ con resección artroscópica de condromatosis sinovial. ${ }^{3,13}$

Frank y colaboradores informaron el caso de un paciente con condromatosis sinovial de codo izquierdo con compresión asintomática del nervio cubital, quien presentaba rigidez y limitación de arcos de movimiento. Realizaron resección abierta por intentos fallidos de resección artroscópica con mejoría clínica. ${ }^{12}$ Mueller y colaboradores reportaron 20 casos de sinovitis condromatosa del codo donde no hubo compresión del nervio cubital y trataron $80 \%$ de los casos con resección artroscópica. ${ }^{14}$

Se han reportado casos de malignización de condromatosis sinovial a condrosarcoma después de la radiosinovectomía. ${ }^{15}$ Existe controversia sobre si la condromatosis sinovial puede malignizar y convertirse en condrosarcoma o si el condrosarcoma es una lesión primaria del sinovio. El condrosarcoma sinovial primario es muy raro y existen pocos reportes en la literatura en inglés. Muramatsu y su equipo informaron un caso de condrosarcoma sinovial del codo en $2012 .{ }^{16}$

\section{CONCLUSIÓN}

Se presentó una paciente con compresión del nervio cubital en el codo por una masa con calcificaciones diagnosticada como condromatosis sinovial, cuya presencia no es habitual en el codo.

La compresión del nervio cubital en el canal epitrócleo olecraneano es la segunda neuropatía compresiva más común en la extremidad superior. La localización más frecuente es en el ligamento de Osborne y la compresión generalmente se da por estructuras anatómicas en el codo. La condromatosis sinovial primaria es una patología benigna y autolimitante. No todos los casos requieren tratamiento quirúrgico, sólo si hay datos clínicos de bloqueo mecánico. Las imágenes radiográficas de la condromato- sis sinovial son altamente sugestivas del diagnóstico y los cirujanos deben familiarizarse con ellas. La resección quirúrgica sin sinovectomía puede ser tratamiento suficiente.

\section{BIBLIOGRAFÍA}

1. Eberlin KR, Marjoua Y, Jupiter JB. Compressive neuropathy of the ulnar nerve: a perspective on history and current controversies. J Hand Surg Am. 2017; 42 (6): 464-469.

2. Earp BE, Floyd WE, Louie D, Koris M, Protomastro P. Ulnar nerve entrapment at the wrist. J Am Acad Orthop Surg. 2014; 22 (11): 699-706.

3. Neumann JA, Garrigues GE, Brigman BE, Eward WC. Synovial chondromatosis. JBJS Rev. 2016; 4 (5). pii: 01874474201605000-00005.

4. Andrews K, Rowland A, Pranjal A, Ebraheim N. Cubital tunnel syndrome: anatomy, clinical presentation, and management. J Orthop. 2018; 15 (3): 832-836. doi: 10.1016/j. jor.2018.08.010.

5. Boone S, Gelberman RH, Calfee RP. The management of cubital tunnel syndrome. J Hand Surg Am. 2015; 40 (9): 18971904; quiz 1904.

6. Cheng CJ, Mackinnon-Patterson B, Beck JL, Mackinnon SE. Scratch collapse test for evaluation of carpal and cubital tunnel syndrome. J Hand Surg Am. 2008; 33 (9): 1518-1524.

7. Davidge KM, Gontre G, Tang D, Boyd KU, Yee A, Damiano MS et al. The "hierarchical" Scratch Collapse Test for identifying multilevel ulnar nerve compression. Hand (N Y). 2015; 10 (3): 388-395.

8. Molinari WJ 3rd, Elfar JC. The double crush syndrome. J Hand Surg Am. 2013; 38 (4): 799-801; quiz 801.

9. Chung KC. Treatment of ulnar nerve compression at the elbow. J Hand Surg Am. 2008; 33 (9): 1625-1627.

10. Foruria AM, Augustin S, Morrey BF, Sánchez-Sotelo J. Heterotopic ossification after surgery for fractures and fracture-dislocations involving the proximal aspect of the radius or ulna. J Bone Joint Surg Am. 2013; 95 (10): e66.

11. Firenstein-Gary S, Budd-Ralph C, Sherine G. Capítulo 128: Tumores y lesiones seudotumorales de las articulaciones y estructuras relacionadas. En: Kelley y Firenstein Tratado de reumatología en dos volúmenes. 10 a edición. Barcelona, España: Ed. Elsevier; 2018. pp. 2073-2075.

12. Milgram JW. J Synovial osteochondromatosis: a histopathological study of thirty cases. Bone Joint Surg Am. 1977; 59 (6): 792-801.

13. de Sa D, Horner NS, MacDonald A, Simunovic N, Ghert MA, Philippon MJ et al. Arthroscopic surgery for synovial chondromatosis of the hip: a systematic review of rates and predisposing factors for recurrence. Arthroscopy. 2014; 30 (11): 1499-1504.e2.

14. Mueller T, Barthel T, Cramer A, Werner A, Gohlke F. Primary synovial chondromatosis of the elbow. J Shoulder Elbow Surg. 2000; 9 (4): 319-322.

15. Sachinis NP, Sinopidis C, Baliaka A, Givissis P. Odyssey of an elbow synovial chondromatosis. Orthopedics. 2015; 38 (1): e62-e67.

16. Muramatsu K, Miyoshi T, Moriya A, Onaka H, Shigetomi M, Nakashima D et al. Extremely rare synovial chondrosarcoma arising from the elbow joint: case report and review of the literature. J Shoulder Elbow Surg. 2012; 21 (2): e7-e11. 